\title{
Construction of Barnes-Wall Lattices from Linear Codes over Rings
}

\author{
J. Harshan \\ Dept. of ECSE, \\ Monash University \\ Clayton, Australia \\ Email:harshan.jagadeesh@monash.edu Email:emanuele.viterbo@monash.edu \\ Emanuele Viterbo \\ Dept. of ECSE, \\ Monash University \\ Clayton, Australia
}

\author{
J.-C. Belfiore \\ Dept. of Communications and Electronics, \\ Telecom ParisTech \\ Paris, France
}

Email:belfiore@enst.fr

\begin{abstract}
Dense lattice packings can be obtained via the wellknown Construction A from binary linear codes. In this paper, we use an extension of Construction A called Construction $A^{\prime}$ to obtain Barnes-Wall lattices from linear codes over polynomials rings. To obtain the Barnes-Wall lattice $B W_{2^{m}}$ in $\mathbb{C}^{2^{m}}$ for any $m \geq 1$, we first identify a linear code $\mathcal{C}_{2}$ over the quotient ring $\mathcal{U}_{m}=\mathbb{F}_{2}[u] / u^{m}$ and then propose a mapping $\psi: \mathcal{U}_{m} \rightarrow$ $\mathbb{Z}[i]$ such that the code $\mathcal{L}_{2^{m}}=\psi\left(\mathcal{C}_{2^{m}}\right)$ is a lattice constellation. Further, we show that $\mathcal{L}_{2}$ has the cubic shaping property when $m$ is even. Finally, we show that $B W_{2^{m}}$ can be obtained through Construction $A^{\prime}$ as $B W_{2^{m}}=(1+i)^{m} \mathbb{Z}[i]^{2^{m}} \oplus \mathcal{L}_{2^{m}}$.
\end{abstract}

\section{INTRODUCTION}

This paper addresses the construction of dense lattice packings [1] using linear block codes [2]. Towards explaining the problem statement, we present a series of definitions used in this paper. A complex lattice $\Lambda$ is a discrete subgroup of $\mathbb{C}^{n}$ [1]. Alternatively, $\Lambda$ is a $\mathbb{Z}[i]$-module generated by the vectors $\left\{\mathbf{v}_{1}, \mathbf{v}_{2}, \cdots, \mathbf{v}_{n} \mid \mathbf{v}_{j} \in \mathbb{C}^{n}\right\}$ as

$$
\Lambda=\left\{\sum_{j=1}^{n} q_{j} \mathbf{v}_{j} \mid \forall q_{j} \in \mathbb{Z}[i]\right\} .
$$

We refer to a set $\mathcal{L}$ as a lattice Euclidean code (or lattice constellation) in $\mathbb{C}^{n}$ if $\mathcal{L}$ is a finite subset of lattice $\Lambda$.

It is well known that any set $\mathcal{S}$ in $\mathbb{C}^{n}$ has a one-one correspondence to a set (denoted by $\overline{\mathcal{S}}$ ) in $\mathbb{R}^{2 n}$ as $\overline{\mathcal{S}}=$ $\{[\Re(\mathbf{x}) \Im(\mathbf{x})] \mid \forall \mathbf{x} \in \mathcal{S}\}$. Using the above equivalence from $\mathbb{C}$ to $\mathbb{R}$, we make the following definition on a lattice Euclidean code.

Definition 1: A lattice Euclidean code $\mathcal{L} \subset \Lambda$ is said to have the cubic shaping property if the corresponding set $\overline{\mathcal{L}}$ can be written as $\overline{\mathcal{L}}=\bar{\Lambda} / a \mathbb{Z}^{2 n}$, for some $a \in \mathbb{Z}$.

The above definition implies that a lattice Euclidean code $\mathcal{L}$ with cubic shaping property is a subset of $\mathbb{Z}_{a}[i]^{n}$ for some $a \in \mathbb{Z}$, where $\mathbb{Z}_{a}$ is the ring of integers modulo $a$. Henceforth, throughout the paper, a Euclidean code refers to a lattice Euclidean code.

Definition 2: (Chapter 4 in [2]) We define the polynomial quotient ring $\mathcal{U}_{m}=\mathbb{F}_{2}[u] / u^{m}$ in variable $u$ for any $m \geq 1$ as

$$
\mathcal{U}_{m}=\left\{\sum_{k=0}^{m-1} b_{k} u^{k} \mid b_{k} \in \mathbb{F}_{2}\right\},
$$

with regular polynomial addition and multiplication over $\mathbb{F}_{2}$ coefficients along with the quotient operation $u^{m}=0$, which is equivalent to cancelling all the terms of degree greater than or equal to $m$.

Definition 3: A subset of $\mathcal{U}_{m}^{n}$ denoted by $\mathcal{C}$ is called a linear code over $\mathcal{U}_{m}$ if $\mathcal{C}$ can be obtained through a generator matrix $\mathbf{G} \in \mathcal{U}_{m}^{k \times n}$ as

$$
\mathcal{C}=\left\{\mathbf{z G} \mid \forall \mathbf{z} \in \mathcal{U}_{m}^{k}\right\},
$$

for some $k \leq n$ and the matrix multiplication is over the ring $\mathcal{U}_{m}$.

Using the above definitions, we now discuss the subject matter of this paper. Systematic use of binary error-correcting codes to construct some structured lattices is well known in the literature [1]. For example, the checkerboard lattice $\mathcal{D}_{2} \subset \mathbb{R}^{2}$ can be constructed as (Chapter 4, Section 7, [1])

$$
\mathcal{D}_{2}=2 \mathbb{Z}^{2} \oplus \mathcal{L}
$$

where $\mathcal{L}=\{\psi(\mathbf{c}) \mid \forall \mathbf{c} \in \mathcal{C}\}$ is an Euclidean code obtained by embedding the codewords of $\mathcal{C}$ the repetition code $(\mathbf{2}, \mathbf{1}, \mathbf{2})$ over $\mathbb{F}_{2}=\{\mathbf{0}, \mathbf{1}\}$ into the Euclidean space using the mapping $\psi: \mathbb{F}_{2} \rightarrow \mathbb{Z}$ such that $\psi(\mathbf{0})=0$ and $\psi(\mathbf{1})=1$. Depending on the structure of the underlying linear error correcting codes, lattice construction can be categorized into different types [1]. In this paper, we consider a special class of constructions called Construction A which is defined formally as follows:

Definition 4: (Sec. 2, Chapter 5, [1]) A lattice $\Lambda$ over $\mathbb{Z}$ is obtained as Construction A from the binary linear code $\mathcal{C}$ if $\Lambda$ can be represented as

$$
\Lambda=2 \mathbb{Z}^{n} \oplus \mathcal{L},
$$

where $\mathcal{L}=\{\psi(\mathbf{c}) \mid \forall \mathbf{c} \in \mathcal{C}\} \subset \mathbb{Z}^{n}$ is an Euclidean code obtained by the component-wise mapping $\psi: \mathbb{F}_{2} \rightarrow \mathbb{Z}$ given by $\psi(\mathbf{0})=0$ and $\psi(\mathbf{1})=1$ on the alphabet of $\mathcal{C}$.

In the above definition, the linear code $\mathcal{C}$ is restricted to be over $\mathbb{F}_{2}$ and $\Lambda$ is viewed as a lattice over $\mathbb{Z}$. However, in general, we could also view $\Lambda$ as a complex lattice, i.e., as a lattice over $\mathbb{Z}[i]$, and construct $\Lambda$ using linear code $\mathcal{C}$ over finite rings. We introduce a new construction of lattices by relaxing the constraint on the alphabet of the linear code $\mathcal{C}$. Our construction is an extension of Construction $\mathrm{A}$ and hence, we refer it as Construction $A^{\prime}$. For the most generalized 
definition of Construction A, we refer the reader to [3]. We use an abstract ring $\mathcal{R}$ to denote either $\mathbb{Z}$ - the ring of integers or $\mathbb{Z}[i]$ - the ring of Gaussian integers.

Definition 5: A lattice $\Lambda$ over $\mathcal{R}$ is obtained through Construction $A^{\prime}$ from the linear code $\mathcal{C}$ over the alphabet $\mathcal{U}_{m}=$ $\mathbb{F}_{2}[u] / u^{m}$ for some $m \geq 1$ if $\Lambda$ can be represented as

$$
\Lambda=u^{m} \mathcal{R}^{n} \oplus \mathcal{L},
$$

where $\mathcal{L}=\{\psi(\mathbf{c}) \mid \forall \mathbf{c} \in \mathcal{C}\} \subset \mathcal{R}^{n}$ is an Euclidean code obtained by using an appropriate mapping $\psi: \mathcal{U}_{m} \rightarrow \mathcal{R}$, and

$$
u=\left\{\begin{array}{c}
2, \text { if } \mathcal{R}=\mathbb{Z}, \\
1+i, \text { if } \mathcal{R}=\mathbb{Z}[i] .
\end{array}\right.
$$

Note that Construction A can be obtained as a special case from Construction $A^{\prime}$ when $m=1$ and $\mathcal{R}=\mathbb{Z}$, wherein the embedding operation $\psi$ coincides with the one in Definition 4.

In this paper, we provide Construction $A^{\prime}$ of Barnes-Wall lattice [1], [4], [5], [6], [7] of dimension $2^{m}$ for $m \geq 1$ by viewing it as a lattice over $\mathbb{Z}[i]$. In other words, we present the following key ingredients needed for Construction $A^{\prime}$ of Barnes-Wall lattices for every $m \geq 1$ :

1) An appropriate linear code $\mathcal{C}_{2^{m}}$ over the alphabet $\mathcal{U}_{m}$.

2) A suitable mapping $\psi: \mathcal{U}_{m} \rightarrow \mathbb{Z}[i]$ in order to obtain the Euclidean code $\mathcal{L}_{2^{m}}$ with cubic shaping property.

Throughout the paper, unless specified, the dimension of the Barnes-Wall lattice refers to its rank as a lattice over $\mathbb{Z}[i]$.

We list the contributions and the organisation of the paper as given below:

- We introduce Construction $A^{\prime}$ of lattices (as in Definition 5) which facilitates us to generate some well structured lattices from linear codes over finite rings. As an immediate application, we apply Construction $A^{\prime}$ to obtain Barnes-Wall lattices of dimension $2^{m}$ by embedding a linear code $\mathcal{C}_{2^{m}}$ over the quotient ring $\mathcal{U}_{m}$ to a Euclidean code in $\mathbb{Z}[i]^{2^{m}}$ for any $m \geq 1$ (Section II)

- First, we identify the structure of the linear code $\mathcal{C}_{2^{m}}$ by using Construction $D$ of Barnes-Wall lattices. Subsequently, we provide a linear encoder to map the information bits onto the codewords of $\mathcal{C}_{2^{m}}$. We identify that the generator matrix for $\mathcal{C}_{2 m}$ is given by

$$
\mathbf{G}_{2^{m}}=\left[\begin{array}{cc}
1 & 1 \\
0 & u
\end{array}\right]^{\otimes m}
$$

where the tensor operation is over $\mathcal{U}_{m}$ (Section II). We also prove the equivalence of our encoding technique to Construction $D$ (Section III).

- To find out $\psi$ and $\mathcal{L}_{2^{m}}$, we first obtain the Euclidean code

$$
\mathcal{E C}_{2^{m}}=\left\{\Phi(\mathbf{c}) \mid \forall \mathbf{c} \in \mathcal{C}_{2^{m}}\right\} \subset \mathbb{Z}[i]^{2^{m}},
$$

through the mapping $\Phi: \mathcal{U}_{m} \rightarrow \mathbb{Z}[i]$ as

$$
\Phi\left(\sum_{j=0}^{m-1} b_{j} u^{j}\right)=\sum_{j=0}^{m-1} b_{j}(\Phi(u))^{j},
$$

with $\Phi(u)=1+i$. At this stage, we point out that $\mathcal{E C}_{2^{m}}$ is an arbitrary subset of $B W_{2^{m}}$ and does not have cubic shaping. To fix this problem, we provide a oneone mapping $\phi: \mathcal{E C}_{2^{m}} \rightarrow \mathcal{L}_{2^{m}} \subset B W_{2^{m}}$ such that $\mathcal{L}_{2^{m}}$ has cubic shaping property for even values of $m$ (Section IV). With this, the mapping $\psi$ (as in Definition 5) is the composition mapping $\phi(\Phi(\cdot))$ on $\mathcal{U}_{m}$, and we show that $B W_{2^{m}}$ is obtained as

$$
B W_{2^{m}}=(1+i)^{m} \mathbb{Z}[i]^{2^{m}} \oplus \mathcal{L}_{2^{m}}
$$

More details on this work can be found in [8]. Apart from providing further details on Construction $A^{\prime}$ of Barnes-Wall lattices, a thorough study on the performance of Euclidean codes from Barnes-Wall lattice constellations is also reported in [8].

Notations: Throughout the paper, boldface letters and capital boldface letters are used to represent vectors and matrices, respectively. For a complex matrix $\mathbf{X}$, the matrices $\mathbf{X}^{T}, \Re(\mathbf{X})$ and $\Im(\mathbf{X})$ denote, respectively, the transpose, real part and imaginary part of $\mathbf{X}$. For a vector $\mathbf{x}$, we use $\mathbf{x}_{j}$ to represent the $j$-th component of $\mathbf{x}$. The set of all integers, the real numbers, and the complex numbers are, respectively, denoted by $\mathbb{Z}, \mathbb{R}$, and $\mathbb{C}$, and $i=\sqrt{-1}$. Cardinality of a set $\mathcal{S}$ is denoted by $|\mathcal{S}|$. Absolute value of a complex number $x$ is denoted by $|x|$. The number of ways of picking $n$ objects out of $m$ objects is denoted by $C_{n}^{m}$. The $n$-length zero vector is denoted by $\mathbf{0}_{n}$

\section{Construction $A^{\prime}$ of Barnes-Wall lattice}

First, we recall Construction $D$ of Barnes-Wall lattices, and subsequently propose its Construction $A^{\prime}$ from a suitable code.

\section{A. Construction D of Barnes-Wall lattice}

Barnes-Wall lattice can be obtained via Construction $D$ [4] as a $\mathbb{Z}[i]$ lattice as follows: Suppose we want to construct the lattice $B W_{2^{m}}$ of dimension $2^{m}$ where $m \geq 1$, let $\mathcal{R} \mathcal{M}(r, m)$ be the binary Reed-Muller (RM) code (Sec. 3.7, Chapter 3, [2]) of length $2^{m}$ and of order $0 \leq r \leq m$. With this, $B W_{2^{m}}$ can be constructed as in (3) given at the top of next page, where $\psi(\cdot)$ is as given in Definition 4. For notational convenience, we also write (3) as

$$
B W_{2^{m}}=(1+i)^{m} \mathbb{Z}[i]^{2^{m}} \oplus \bigoplus_{r=0}^{m-1}(1+i)^{r} \mathcal{R} \mathcal{M}(r, m) .
$$

This method generates $B W_{2^{m}}$ as a multi-level structure of nested RM codes and hence it falls under Construction $D$ [1].

Example 1: $B W_{4}$ (which is also known as $E_{8}$ over $\mathbb{Z}$ ) is constructed as

$$
B W_{4}=(1+i)^{2} \mathbb{Z}[i]^{4} \oplus(1+i) \mathcal{R} \mathcal{M}(1,2) \oplus \mathcal{R} \mathcal{M}(0,2),
$$

where the code $\mathcal{R} \mathcal{M}(1,2)=(\mathbf{4}, \mathbf{3}, \mathbf{2})$ and the code $\mathcal{R} \mathcal{M}(0,2)=(\mathbf{4}, \mathbf{1}, \mathbf{4})$ in the classical $\left(\mathbf{n}, \mathbf{k}, \mathbf{d}_{\mathbf{m i n}}\right)$ format.

Example 2: $B W_{16}$ is obtained as

$$
\begin{array}{r}
B W_{16}=(1+i)^{4} \mathbb{Z}[i]^{16} \oplus(1+i)^{3}(\mathbf{1 6}, \mathbf{1 5}, \mathbf{2}) \oplus \\
(1+i)^{2}(\mathbf{1 6}, \mathbf{1 1}, \mathbf{4}) \oplus(1+i)(\mathbf{1 6}, \mathbf{5 , ~ 8 )} \oplus(\mathbf{1 6}, \mathbf{1 , ~ 6 ) .}
\end{array}
$$




$$
B W_{2^{m}}=\left\{(1+i)^{m} \mathbf{a}+\sum_{r=0}^{m-1}(1+i)^{r} \psi\left(\mathbf{c}_{r}\right) \mid \forall \mathbf{c}_{r} \in \mathcal{R} \mathcal{M}(r, m), \forall \mathbf{a} \in \mathbb{Z}[i]^{2^{m}}\right\}
$$

\section{B. Construction $A^{\prime}$}

In order to obtain $B W_{2^{m}}$ as Construction $A^{\prime}$, we first need to find a suitable linear code $\mathcal{C}_{2^{m}}$ over an appropriate ring. To find such a code, we are interested in understanding the following expression in (4),

$$
\mathcal{E C}_{2^{m}}=\bigoplus_{r=0}^{m-1}(1+i)^{r} \mathcal{R} \mathcal{M}(r, m)
$$

as a single code. If we denote $u=1+i$ and consider $u$ as a symbol, then the expression

$$
\sum_{r=0}^{m-1} u^{r} \mathcal{R} \mathcal{M}(r, m)
$$

can be viewed as a code denoted by $\mathcal{C}_{2^{m}}$ over the ring $\mathcal{U}_{m}$.

Example 3: For $B W_{4}$, the code $\mathcal{C}_{4}$ is given by $u(\mathbf{4}, \mathbf{3}, \mathbf{2})+$ $(4,1,4)$, which can be viewed as a code over the quotient ring $\mathcal{U}_{2}$.

Example 4: Another example is $\mathcal{C}_{16}$, which is obtained from $B W_{16}$ and is given by

$\mathcal{C}_{16}=(\mathbf{1 6}, \mathbf{1}, \mathbf{6})+u(\mathbf{1 6}, \mathbf{5}, \mathbf{8})+u^{2}(\mathbf{1 6}, \mathbf{1 1}, \mathbf{4})+u^{3}(\mathbf{1 6}, \mathbf{1 5}, \mathbf{2})$,

where $\mathcal{C}_{16}$ is defined over $\mathcal{U}_{4}$.

In general, the ring on which the code

$$
\mathcal{C}_{2^{m}}=\sum_{r=0}^{m-1} u^{r} \mathcal{R} \mathcal{M}(r, m)
$$

is defined is the quotient ring $\mathcal{U}_{m}$. With this, we have identified the linear code $\mathcal{C}_{2^{m}}$ to be useful for Construction $A^{\prime}$ of $B W_{2^{m}}$.

In the rest of this subsection, we provide a linear encoder to generate the codewords of $\mathcal{C}_{2^{m}}$. It is known that the $2^{m}$ dimensional Barnes-Wall lattice $B W_{2^{m}}$ over $\mathbb{Z}[i]$ is generated by the rows of the $m$-fold Kronecker product [6]

$$
\mathbf{G}=\left[\begin{array}{cc}
1 & 1 \\
0 & (1+i)
\end{array}\right]^{\otimes m}
$$

Replacing $u=1+i$ as a symbol and making $u^{m}=0$ in $\mathbf{G}$, we obtain the generator matrix $\mathbf{G}_{2^{m}}$ which can be viewed as a matrix over $\mathcal{U}_{m}$.

Example 5: The generator matrix $\mathbf{G}_{4}$ is given by

$$
\mathbf{G}_{4}=\left[\begin{array}{cccc}
1 & 1 & 1 & 1 \\
0 & u & 0 & u \\
0 & 0 & u & u \\
0 & 0 & 0 & 0
\end{array}\right] \in \mathcal{U}_{2}^{4 \times 4}
$$

By using $\mathbf{G}_{2^{m}}$ as a matrix over $\mathcal{U}_{m}$, the code $\mathcal{C}_{2^{m}}$ is obtained as below:
Encoding of $\mathcal{C}_{2^{m}}$ : Let $\mathbf{z} \in \mathcal{U}_{m}^{2^{m}}$, i.e., the $j$-th component of $\mathbf{z}$ is given by

$$
\mathbf{z}_{j}=\sum_{k=0}^{m-1} b_{k, j} u^{k}
$$

where $b_{k, j} \in \mathbb{F}_{2}$ for all $k, j$. Using $\mathbf{z}$ and $\mathbf{G}_{2^{m}}$, the code $\mathcal{C}_{2^{m}} \subset \mathcal{U}_{m}^{2^{m}}$ can be obtained as

$$
\mathcal{C}_{2^{m}}=\left\{\mathbf{x}=\mathbf{z G}_{2^{m}} \mid \forall \mathbf{z} \in \mathcal{U}_{m}^{2^{m}}\right\},
$$

where the matrix multiplication is over $\mathcal{U}_{m}$.

Proposition 1: The rate of the code $\mathcal{C}_{2^{m}}$ in bits per codeword is $\left(\frac{m}{2}\right) 2^{m}$.

Proof: Each component of $\mathbf{z}$ carries $m$ information bits in the variables $b_{k, j}$ as shown in (8). This amounts to a total of $m 2^{m}$ bits carried by $\mathbf{z}$. However, since the matrix multiplication is over $\mathcal{U}_{m}$, not all the information bits $b_{k, j}$ are encoded as codewords of $\mathcal{C}_{2^{m}}$ (due to $u^{m}=0$ ). Using the structure of $\mathbf{G}_{2^{m}}$ it is possible to identify the indices $(k, j)$ of information bits $b_{k, j}$ which get encoded into the codewords of $\mathcal{C}_{2^{m}}$ as follows: Let the set $\mathcal{I}_{q}$ denote the indices of the rows of $\mathbf{G}_{2^{m}}$ whose components take values from the binary set $\left\{0, u^{q}\right\}$ for $q=0,1, \cdots m-1$. Due to the quotient operation $u^{m}=0$, the components of $\mathbf{z}$ which are in the index set $\mathcal{I}_{q}$ are restricted to be of the form, $\mathbf{z}_{j}=\sum_{k=0}^{m-1-q} b_{k, j} u^{k} \forall j \in \mathcal{I}_{q}$. For example, $\mathbf{z}_{1}=\sum_{k=0}^{m-1} b_{k, 1} u^{k}$ and $\mathbf{z}_{2^{m}}=0$. Using the structure of $\mathbf{G}_{2^{m}}$ we observe that $\left|\mathcal{I}_{q}\right|=C_{q}^{m}$, and hence find the total number of information bits on a codeword of $\mathcal{C}_{2^{m}}$ as $\sum_{k=0}^{m}(m-k) C_{k}^{m}$. Therefore, the rate of $\mathcal{C}_{2^{m}}$ in bits per codeword is $\sum_{i=0}^{m-1} C_{i}^{m}(m-i)=\frac{m}{2} 2^{m}$.

We now provide an example for the proposed encoding technique, showcasing the positions of the information bits that get encoded to the codewords of $\mathcal{C}_{2^{m}}$.

Example 6: For $m=3$, the input vector $\mathbf{z}$ and $\mathbf{G}_{8}$ are of the form,

$$
\mathbf{z}^{T}=\left[\begin{array}{c}
b_{0,1}+b_{1,1} u+b_{2,1} u^{2} \\
b_{0,2}+b_{1,2} u \\
b_{0,3}+b_{1,3} u \\
b_{0,4} \\
b_{0,5}+b_{1,5} u \\
b_{0,6} \\
b_{0,7} \\
0
\end{array}\right] \text { and }
$$

$$
\mathbf{G}_{8}=\left[\begin{array}{cccccccc}
1 & 1 & 1 & 1 & 1 & 1 & 1 & 1 \\
0 & u & 0 & u & 0 & u & 0 & u \\
0 & 0 & u & u & 0 & 0 & u & u \\
0 & 0 & 0 & u^{2} & 0 & 0 & 0 & u^{2} \\
0 & 0 & 0 & 0 & u & u & u & u \\
0 & 0 & 0 & 0 & 0 & u^{2} & 0 & u^{2} \\
0 & 0 & 0 & 0 & 0 & 0 & u^{2} & u^{2} \\
0 & 0 & 0 & 0 & 0 & 0 & 0 & 0
\end{array}\right] .
$$




\section{On Equivalence of Construction $A^{\prime}$ TO CONSTRUCTION $D$}

In this subsection, we prove the equivalence of our encoding technique to Construction $D$. In other words, the following theorem shows that the codewords generated in (9) can be uniquely represented as vectors of a multi-level code of nested RM codes as in (7).

Theorem 1: The codewords generated in (9) can be uniquely represented as codewords obtained through Construction $D$.

Proof: The entries of $\mathbf{G}_{2^{m}}$ take values from the set $\left\{0,1, u, u^{2}, \cdots u^{m-1}\right\}$. After suitable row permutations, $\mathbf{G}_{2^{m}}$ can be written as

$$
\mathbf{G}_{2^{m}}=\left[\begin{array}{c}
\mathbf{R}_{0} \\
u \mathbf{R}_{1} \\
\vdots \\
u^{m-1} \mathbf{R}_{m-1} \\
u^{m} \mathbf{R}_{m}
\end{array}\right]
$$

where $\mathbf{R}_{k} \in \mathbb{F}_{2}^{C_{k}^{m} \times 2^{m}}$. Note that $\left[\begin{array}{llll}\mathbf{R}_{0}^{T} & \mathbf{R}_{1}^{T} & \cdots & \mathbf{R}_{r}^{T}\end{array}\right]^{T}$ is a generator matrix of the $r$-th order $\mathrm{RM}$ code for $r \leq m$. Recalling the encoding technique, the code $\mathcal{C}_{2^{m}}$ is obtained as

$$
\mathcal{C}_{2^{m}}=\left\{\mathbf{x}=\mathbf{z G}_{2^{m}} \mid \forall \mathbf{z} \in \mathcal{U}_{m}^{2^{m}}\right\}
$$

where the matrix multiplication is over $\mathcal{U}_{m}$. The vector $\mathbf{z}$ can be written as $\mathbf{z}=\mathbf{u B}$, where

$$
\begin{aligned}
& \mathbf{u}=\left[\begin{array}{llllll}
1 & u & u^{2} & \cdots & u^{m-2} & u^{m-1}
\end{array}\right] \in \mathcal{U}_{m}^{1 \times m} \\
& \mathbf{B}=\left[\begin{array}{ccccc}
b_{0,0} & b_{0,1} & \cdots & b_{0,2^{m}-2} & b_{0,2^{m}-1} \\
b_{1,0} & b_{1,1} & \cdots & b_{1,2^{m}-2} & b_{1,2^{m}-1} \\
b_{2,0} & b_{2,1} & \cdots & b_{2,2^{m}-2} & b_{2,2^{m}-1} \\
\vdots & \vdots & \cdots & \vdots & \vdots \\
b_{m-2,0} & b_{m-2,1} & \cdots & b_{m-2,2^{m}-2} & b_{m-2,2^{m}-1} \\
b_{m-1,0} & b_{m-1,1} & \cdots & b_{m-1,2^{m}-2} & b_{m-1,2^{m}-1}
\end{array}\right] \in \mathbb{F}_{2}^{m \times 2^{m}}
\end{aligned}
$$

Note that $b_{k, j}$ are the information bits to be encoded into codewords of $\mathcal{C}_{2^{m}}$. We split the information matrix $\mathbf{B}$ as $\left[\begin{array}{llll}\mathbf{B}_{0} & \mathbf{B}_{1} & \cdots & \mathbf{B}_{m}\end{array}\right]$ where $\mathbf{B}_{k} \in \mathbb{F}_{2}^{m \times C_{k}^{m}}$ for $k=0,1, \cdots m$. After the above split, the BW lattice vector $\mathbf{x}$ is obtained as

$$
\mathbf{x}=\mathbf{u}\left[\begin{array}{llll}
\mathbf{B}_{0} & \mathbf{B}_{1} & \cdots & \mathbf{B}_{m}
\end{array}\right]\left[\begin{array}{c}
\mathbf{R}_{0} \\
u \mathbf{R}_{1} \\
\vdots \\
u^{m-1} \mathbf{R}_{m-1} \\
u^{m} \mathbf{R}_{m}
\end{array}\right] .
$$

The R.H.S of the above operation can be alternately written as

$$
\mathbf{x}=\mathbf{u}\left[\begin{array}{llll}
\overline{\mathbf{B}}_{0} & \overline{\mathbf{B}}_{1} & \cdots & \overline{\mathbf{B}}_{m}
\end{array}\right] \underbrace{\left[\begin{array}{c}
\mathbf{R}_{0} \\
\mathbf{R}_{1} \\
\vdots \\
\mathbf{R}_{m-1} \\
\mathbf{R}_{m}
\end{array}\right]}_{\mathbf{G}_{R M}},
$$

where $\overline{\mathbf{B}}_{k}=\left[\begin{array}{c}\mathbf{0}_{k \times C_{k}^{m}} \\ \mathbf{B}_{k}([1: m-k],:)\end{array}\right]$. Note that $\mathbf{G}_{R M}$ is the nested RM generator matrix. We use the notation $\overline{\mathbf{B}}=$ $\left[\begin{array}{llll}\overline{\mathbf{B}}_{0} & \overline{\mathbf{B}}_{1} & \cdots & \overline{\mathbf{B}}_{m}\end{array}\right]$. We also point out that the informations bits in each row of $\overline{\mathbf{B}}$ are encoded to RM codewords of appropriate order by the matrix multiplication $\overline{\mathbf{B}} \mathbf{G}_{R M}$. Due to zero entries in $\overline{\mathbf{B}}$, the matrix $\overline{\mathbf{B}}$ has only $\sum_{n=0}^{k-1} C_{n}^{m}$ information bits in the $k$-th row of $\overline{\mathbf{B}}$ for $k=1,2, \cdots m$. Since, these $\sum_{n=0}^{k-1} C_{n}^{m}$ bits are placed in the first as many columns of $\overline{\mathbf{B}}$, the information bits in the $k$-th row of $\overline{\mathbf{B}}$ are encoded into a RM codeword of $(k-1)$-th order. Finally, on the multiplication of $\mathbf{u}$ from left, the generated RM codewords are appropriately weighed by powers of $u$ and added. This proves the equivalence of our construction to Construction $D$.

Till now, we have identified the linear code $\mathcal{C}_{2^{m}}$ and its encoding technique over the quotient ring $\mathcal{U}_{m}$. In the next subsection, we discuss embedding of $\mathcal{C}_{2^{m}}$ into the Euclidean space $\mathbb{Z}[i]^{2^{m}}$.

\section{EMBEDDING to BARNES-WALl LATTICE AND CUBIC SHAPING}

By using the map $\Phi(u)=1+i$ on $\mathcal{C}_{2^{m}}$, we get a Euclidean code given by

$$
\begin{aligned}
\mathcal{E C}_{2^{m}} & =\left\{\Phi(\mathbf{c}) \mid \forall \mathbf{c} \in \mathcal{C}_{2^{m}}\right\} \in \mathbb{Z}[i]^{2^{m}}, \\
& =\bigoplus_{r=0}^{m-1}(1+i)^{r} \mathcal{R} \mathcal{M}(r, m),
\end{aligned}
$$

where $\Phi$ maps the symbols of $\mathcal{U}_{m}$ into $\mathbb{Z}[i]$ as

$$
\Phi\left(\sum_{j=0}^{m-1} b_{j} u^{j}\right)=\sum_{j=0}^{m-1} b_{j}(\Phi(u))^{j} .
$$

It is to be noted that $\mathcal{E C}_{2^{m}}$ is an arbitrary subset of $B W_{2^{m}}$ and does not have cubic shaping. To fix this problem, we propose a one-one mapping $\phi$ on $\mathcal{E C}_{2^{m}}$ to obtain a new Euclidean code denoted by $\mathcal{L}_{2^{m}}$ such that $\mathcal{L}_{2^{m}}$ has cubic shaping when $m$ is even. For any $\mathbf{x}=\left[x_{1}, x_{2}, x_{3}, \cdots, x_{2^{m}}\right] \in \mathcal{E C}_{2^{m}}$, the mapping $\phi$ operates on each component of $\mathbf{x}$ as,

$$
\phi\left(x_{j}\right)=\left\{\begin{array}{c}
x_{j} \bmod 2^{\frac{m}{2}}, \text { when } m \text { is even; } \\
\varphi\left(x_{j} \bmod 2^{\frac{m+1}{2}}\right), \text { when } m \text { is odd, }
\end{array}\right.
$$

where $\varphi(\cdot)$ is defined on $\mathbb{Z}_{2 \frac{m+1}{2}}[i]$ as,

$$
\varphi(z)=\left\{\begin{array}{c}
z, \text { when } \Im(z)<2^{\frac{m-1}{2}} \\
z+\left(2^{\frac{m-1}{2}}-i 2^{\frac{m-1}{2}}\right), \text { when } \Re(z)<2^{\frac{m-1}{2}} \\
\text { and } \Im(z) \geq 2^{\frac{m-1}{2}} \\
z-\left(2^{\frac{m-1}{2}}+i 2^{\frac{m-1}{2}}\right), \text { when } \Re(z) \geq 2^{\frac{m-1}{2}} \\
\text { and } \Im(z) \geq 2^{\frac{m-1}{2}} .
\end{array}\right.
$$

The mapping $\phi$ guarantees the following property on $\mathcal{L}_{2^{m}}$ :

$\mathcal{L}_{2^{m}} \subset\left\{\begin{array}{c}\left\{\mathbb{Z}_{2^{\frac{m}{2}}}[i]\right\}^{2^{m}}, \text { if } m \text { is even; } \\ \left\{\mathbb{Z}_{2^{\frac{m+1}{2}}}\right\}^{2^{m}}+i\left\{\mathbb{Z}_{2^{\frac{m-1}{2}}}\right\}^{2^{m}}, \text { if } m \text { is odd. }\end{array}\right.$ 
From (15), note that each component of the vector in $\mathcal{L}_{2^{m}}$ is in a cubic box and a rectangular box, when $m$ is even and odd, respectively. With this, the mapping $\psi$ (given in Definition 5) needed to obtain the Euclidean code $\mathcal{L}_{2^{m}}$ from $\mathcal{C}_{2^{m}}$ can be written as

$$
\psi=\phi(\Phi(\cdot))
$$

where $\Phi$ and $\phi$ are given in (12) and (13) respectively.

Proposition 2: The rate of the Euclidean code $\mathcal{L}_{2^{m}}$ in bits per complex dimension is $\frac{m}{2}$.

Proof: The proof follows from the one-one nature of $\psi$ and the result of Proposition 1. For the proof on the one-one nature of $\psi$, we refer the reader to Proposition 2 of [8].

Remark 1: We point out that Construction $A^{\prime}$ does not qualify to be the generalized Construction A of [3] since the proposed embedding operation $\psi$ is not a linear map.

The following theorem shows that $\psi$ retains the BarnesWall lattice structure on $\mathcal{L}_{2^{m}}$ and proves Construction $A^{\prime}$ of $B W_{2^{m}}$.

Theorem 2: The Euclidean code $\mathcal{L}_{2^{m}}$ and the lattice $B W_{2^{m}}$ are related as $B W_{2^{m}}=(1+i)^{m} \mathbb{Z}[i]^{2^{m}} \oplus \mathcal{L}_{2^{m}}$.

Proof: Consider the case when $m$ is even. From (3) and (11), any $\mathbf{z} \in B W_{2^{m}}$ can be written as

$$
\mathbf{z}=(1+i)^{m} \mathbf{a}+\mathbf{x}
$$

where $\mathbf{a} \in \mathbb{Z}[i]^{2^{m}}$ and $\mathbf{x} \in \mathcal{E C}_{2^{m}}$. Further, upon the modulo operation in (13), $\mathbf{x}$ satisfies $\mathbf{x}=2^{\frac{m}{2}} \mathbf{r}+\phi(\mathbf{x})$, where $\phi(\mathbf{x}) \in$ $\mathcal{L}_{2^{m}}$ and $\mathbf{r} \in \mathbb{Z}[i]^{2^{m}}$. This implies

$$
\phi(\mathbf{x})=\mathbf{x}-2^{\frac{m}{2}} \mathbf{r}=\mathbf{x}+(1+i)^{m} \mathbf{r}^{\prime},
$$

for some $\mathbf{r}^{\prime} \in \mathbb{Z}[i]^{2^{m}}$. The second equality follows as

$$
(1+i)^{m}=a 2^{\frac{m}{2}} \text { where } a \in\{1,-1, i,-i\} .
$$

The R.H.S of (18) is in the form of (3) and hence $\mathcal{L}_{2^{m}} \subset$ $B W_{2^{m}}$. Further, combining (17) and (18), we have

$$
\mathbf{z}=(1+i)^{m} \mathbf{a}^{\prime}+\phi(\mathbf{x}),
$$

for some $\mathbf{a}^{\prime} \in \mathbb{Z}[i]^{2^{m}}$ and $\phi(\mathbf{x}) \in \mathcal{L}_{2^{m}}$. From (15), we also observe that

$$
(1+i)^{m} \mathbb{Z}[i]^{2^{m}} \cap \mathcal{L}_{2^{m}}=2^{\frac{m}{2}} \mathbb{Z}[i]^{2^{m}} \cap \mathcal{L}_{2^{m}}=\left\{\mathbf{0}_{2^{m}}\right\} .
$$

The first equality in the above equation follows from (19). With (20) and (21), the statement of the theorem follows when $m$ is even.

We now consider the case when $m$ is odd. For this case, we first study the mod $2^{\frac{m+1}{2}}$ operation in (13), and subsequently study the effect of $\varphi$. With the mod operation, any $\mathbf{x} \in \mathcal{E C}_{2^{m}}$ satisfies $\mathbf{x}=2^{\frac{m+1}{2}} \mathbf{r}+\overline{\mathbf{x}}$, where $\overline{\mathbf{x}} \in \mathbb{Z}_{2^{\frac{m+1}{2}}}[i]^{2^{m}}$ and $\mathbf{r} \in$ $\mathbb{Z}[i]^{2^{m}}$. This implies

$$
\overline{\mathbf{x}}=\mathbf{x}-2^{\frac{m+1}{2}} \mathbf{r}=\mathbf{x}+(1+i)^{m} \mathbf{r}^{\prime},
$$

for some $\mathbf{r}^{\prime} \in \mathbb{Z}[i]^{2^{m}}$. The second equality follows as $2^{\frac{m+1}{2}}=$ $a \cdot(1+i)^{m}$ for some $a \in \mathbb{Z}[i]$. We point out that $\overline{\mathbf{x}}$ is already a
Barnes-Wall lattice point. Further, the constants added in (14) are such that

$$
2^{\frac{m-1}{2}}(1-i)=a \cdot(1+i)^{m} \text { and } 2^{\frac{m-1}{2}}(1+i)=b \cdot(1+i)^{m}
$$

for some $a, b \in \mathbb{Z}[i]$. Therefore, $\varphi(\overline{\mathbf{x}})$ continues to be a BarnesWall lattice point. We also know that $\mathbf{x}=(1+i)^{m} \mathbf{r}+\phi(\mathbf{x})$, for some $\mathbf{r} \in \mathbb{Z}[i]^{2^{m}}$ and $\phi(\mathbf{x}) \in \mathcal{L}_{2^{m}}$. Finally, from (15), we have

$(1+i)^{m} \mathbb{Z}[i]^{2^{m}} \cap \mathcal{L}_{2^{m}}=2^{\frac{m-1}{2}}(1+i) \mathbb{Z}[i]^{2^{m}} \cap \mathcal{L}_{2^{m}}=\left\{\mathbf{0}_{2^{m}}\right\}$.

The first equality in the above equation follows as $(1+i)^{m}$ is of the form $a 2^{\frac{m-1}{2}}$ where $a= \pm 1 \pm i$. This completes the proof when $m$ is odd.

Using the results of Theorem 2, the Construction $A^{\prime}$ of $B W_{2^{m}}$ is given by $B W_{2^{m}}=(1+i)^{m} \mathbb{Z}[i]^{2^{m}} \oplus \mathcal{L}_{2^{m}}$, where $\mathcal{L}_{2^{m}}$ is the Euclidean code obtained from $\mathcal{C}_{2^{m}}$ through the mapping $\psi=\phi(\Phi(\cdot))$ on $\mathcal{U}_{m}$.

\section{CONCLUSION}

In this paper, we have introduced an extension of Construction A to obtain the class of Barnes-Wall lattices from linear codes over rings. We highlight that the mapping $\psi$ provides the cubic shaping property on the Euclidean code, which in turn facilitates labelling of information bits if the Euclidean code is to be used as a coded modulation scheme.

\section{ACKNOWLEDGMENT}

This work was performed within the Monash Software Defined Telecommunications Lab and supported by the Monash Professional Fellowship 2011.

\section{REFERENCES}

[1] J.H. Conway and N.J.A Sloane, Sphere Packings, Lattices and Groups, Second Edition, 1993, Springer-Verlag, New York.

[2] R. E. Blahut, Theory and Practice of Error Control Codes, AddisonWesley Publishing Company, Inc, 1983.

[3] G.D. Forney, and A. Vardy, "Generalized Minimum-Distance Decoding of Euclidean-Space Codes and Lattices", IEEE Transactions on Information Theory, vol. 42, no. 6, Nov. 1996, pp. 1992 - 2026.

[4] G.D. Forney, "Coset Codes- Part II: Binary Lattices and Related Codes", IEEE Transactions on Information Theory, vol. 34, no. 5, Sept. 1988, pp. 1152 - 1187

[5] G. Nebe, E. M. Rains and N. J. A. Sloane, "A Simple Construction of the Barnes-Wall Lattices", in Codes, Graphs, and Systems: A Celebration of the Life and Career of G. David Forney, Jr. on the Occasion of his Sixtieth Birthday, ed. R. E. Blahut and R. Koetter, Kluwer, 2002, pp. 333-342.

[6] D. Micciancio, and A. Nicolsi, "Efficient Bounded Distance Decoders for Barnes-Wall Lattices", in the Proc. of IEEE ISIT 2008, Toronto, Canada, July 6-11, 2008.

[7] A. J. Salomon, and O. Amrani, "Augmented Product Codes and Lattices: Reed-Muller Codes and Barnes-Wall Lattices", IEEE Transactions on Information Theory, vol. 51, no. 11, Nov. 2005, pp. 3918 - 3930.

[8] J. Harshan, E. Viterbo, and J.-C. Belfiore, "Practical Encoders and Decoders for Euclidean Codes from Barnes-Wall Lattices," available on arXiv:1203.3282v2 [cs.IT], March 2012. 